EM tomographic image reconstruction using polar voxels

This article has been downloaded from IOPscience. Please scroll down to see the full text article. 2013 JINST 8 C01004

(http://iopscience.iop.org/1748-0221/8/01/C01004)

View the table of contents for this issue, or go to the journal homepage for more

Download details:

IP Address: 158.42.104.5

The article was downloaded on 07/01/2013 at 09:18

Please note that terms and conditions apply. 


\title{
EM tomographic image reconstruction using polar voxels
}

\author{
A. Soriano, ${ }^{a, 1}$ M.J. Rodríguez-Alvarez, ${ }^{a}$ A. Iborra,,${ }^{b}$ F. Sánchez,${ }^{a}$ M. Carles, ${ }^{a}$ \\ P. Conde ${ }^{a}$ A.J. González, ${ }^{a}$ L. Hernández, ${ }^{a}$ L. Moliner, ${ }^{a}$ A. Orero, ${ }^{a}$ L.F. Vidal ${ }^{a}$ \\ and J.M. Benlloch ${ }^{a}$ \\ ${ }^{a}$ Instituto de Instrumentación para Imagen Molecular (I3M), Centro Mixto CSIC-UPV-CIEMAT, \\ Camino de Vera s/n., (E-46022) Valencia, Spain \\ ${ }^{b}$ Instituto de Matemática Multidisciplinar, Universitat Politécnica de València, \\ Camino de Vera s/n., (E-46022) Valencia, Spain \\ E-mail: asoriano@i3m.upv.es
}

ABSTRACT: The splitting of the field of view (FOV) in polar voxels is proposed in this work in order to obtain an efficient description of a cone-beam computed tomography (CT) scanner. The proposed symmetric-polar pixelation makes it possible to deal with the 3D iterative reconstruction considering a number of projections and voxel sizes typical in CT preclinical imaging.

The performance comparison, between the filtered backprojection (FBP) and 3D maximum likelihood expectation maximization (MLEM) reconstruction algorithm for CT, is presented. It is feasible to achieve the hardware spatial resolution limit with the considered pixelation. The image quality achieved with MLEM and FBP have been analyzed. The results obtained with both algorithms in clinical images have been compared too. Although the polar-symmetric pixelation is presented in the context of CT imaging, it can be applied to any other tomographic technique as long as the scan comprises the measurement of an object under several projection angles.

KEYWORDS: Image reconstruction in medical imaging; Data reduction methods

\footnotetext{
${ }^{1}$ Corresponding author.
} 


\section{Contents}

1 Introduction 1

2 System matrix 1

3 Experimental results $\quad 2$

4 Conclusion 5

\section{Introduction}

Analytical methods like filtered backprojection (FBP) [1] have dominated the image reconstruction in computed tomography $(\mathrm{CT})$ because they produce images of a reasonable quality with low cost in terms of computing time. Current advances in computer science enable the use of iterative methods like maximum likelihood expectation maximization (MLEM) [2] in CT image reconstruction [3, 4]. The improvement of image quality with iterative methods allows to reduce the dose a patient receives during a CT examination as much as a $63 \%$ [5].

The main drawback of iterative methods is the large system matrices required for a precise description of the scanner. The authors have already presented in [6] a description of the CT scanner in polar coordinates that allows to reduce drastically the number of matrix elements to be computed. Several approaches were studied in [6] to discretize the field of view (FOV) in polar coordinates in a fan beam CT geometry. The approach proposed in [6] has been extended to cone beam geometry and its performance is compared in this work with that obtained with the FBP, based on measurements of synthetic phantoms and clinical images acquired with a preclinical CT scanner.

\section{System matrix}

Iterative methods consider the reconstruction problem as a linear system $\hat{\varphi}=P \psi$. The tomograph is modeled with the probability matrix $P$ which links the reconstructed attenuation map $\psi$ with the estimation of the measurement $\hat{\varphi}$. Each matrix element $P_{i j}$ represents the contribution of the $\mathrm{j}$-th voxel to the attenuation of the i-th ray. Siddon's algorithm [7] is the most common approach to determine the system matrix. However, the intersection volume between the voxel and the ray was considered to calculate the matrix elements, as it is described by Yao et al. in [8]. The large number of projections and high resolutions $(\sim 0.1 \mathrm{~mm})$ used in CT lead to huge linear systems. The description of the scanner in polar coordinates only requires the calculation of the matrix elements of the first projection, while the remaining elements in $P$ are obtained by rotating those in the first projection. The strong reduction in matrix size when considering a polar discretization is shown in table 1. From table 1 it can be inferred that the matrix size obtained with a cartesian discretization 
Table 1. Matrix sizes and reconstruction times using cartesian and polar discretizations of the FOV.

\begin{tabular}{|ccccccc|}
\hline & \multicolumn{3}{c}{ Cartesian } & \multicolumn{3}{c|}{ Polar } \\
Projections & $\begin{array}{c}\text { Size } \\
\end{array}$ & \multicolumn{2}{c|}{ Time (s) } & Size & \multicolumn{2}{c|}{ Time (s) } \\
& $(\mathrm{MB})$ & Generation & Iteration & $(\mathrm{MB})$ & Generation & Iteration \\
\hline 40 & 486 & 43 & 0.6 & 52 & 5 & 0.5 \\
80 & 972 & 69 & 1 & 57 & 5.4 & 1.2 \\
100 & 1200 & 88 & 1.4 & 60 & 5.7 & 1.8 \\
200 & 2400 & 161 & 3 & 75 & 7.1 & 6.5 \\
400 & 4800 & 318 & 5.8 & 104 & 10 & 25.8 \\
\hline
\end{tabular}

of the FOV rises about 80 times faster with the number of projections than that obtained in the polar case. The increase in the polar case is partly due to the oversampling of the central region of the FOV, which leads to differences in reconstruction times. The values in table 1 correspond to a $80 \mathrm{~mm}$ FOV discretized in cubic voxels of $0.8 \mathrm{~mm}$. Considering a typical CT scan with 200 projections, the reduction of the voxel size to $0.4 \mathrm{~mm}$ requires $8.5 \mathrm{~GB}$ to store the system matrix when a cartesian discretization of the FOV is considered. But only the $224 \mathrm{MB}$ of the first projection are required in the polar case, because the full matrix is constructed as a block circulant matrix. The large system matrices required for a cartesian discretization hinders the use of iterative algorithms in typical CT acquisitions having 200 projections and voxel sizes of $0.4 \mathrm{~mm}$. Such a limitation can be overcome thanks to the small matrix sizes required with the polar discretization.

\section{Experimental results}

Experimental measurements were conducted with the Albira $\mu \mathrm{CT}$, manufactured by Oncovision [9]. It consists of a $35 \mu \mathrm{m}$ focal spot size $\mathrm{X}$ ray tube, with a variable voltage from $10 \mathrm{kV}$ to $50 \mathrm{kV}$. The detector is a CsI flat panel with $2400 \times 2400$ pixels of $50 \mu \mathrm{m}$. X ray tube and detector are mounted in a cone-beam configuration having a FOV of $80 \mathrm{~mm}$ in diameter and $90 \mu \mathrm{m}$ spatial resolution.

The number of iterations considered in the reconstruction influences the performance of MLEM. In order to facilitate the comparison among the scenarios evaluated in this work, a fixed number of 40 iterations that ensures the convergence of the algorithm, was employed in all reconstructions.

The spatial resolution performance of the FBP and MLEM algorithms has been assessed through the comparison of the modulation transfer function (MTF) curves. They have been calculated as the Fourier transform of the reconstructed images [10] of a $50 \mu \mathrm{m}$ gold wire inside a cylinder, $20 \mathrm{~mm}$ in diameter, polymethyl methacrylate (PMMA) phantom. The reduction of the voxel size from $30 \mu \mathrm{m}$ (figure 1(a)) to $5 \mu \mathrm{m}$ (figure 1(b)) leads to an improvement in the spatial frequency at $10 \%$ of the MTF from $10.3 \mathrm{lp} \mathrm{mm}^{-1}$ to $11.2 \mathrm{lp} \mathrm{mm}^{-1}$ when the MLEM algorithm has been considered, and from $7.01 \mathrm{p} \mathrm{mm}^{-1}$ to $11.2 \mathrm{lp} \mathrm{mm}^{-1}$ if the FBP is chosen. No further enhancement of the spatial resolution has been observed when voxel size's smaller than $5 \mu \mathrm{m}$ were 


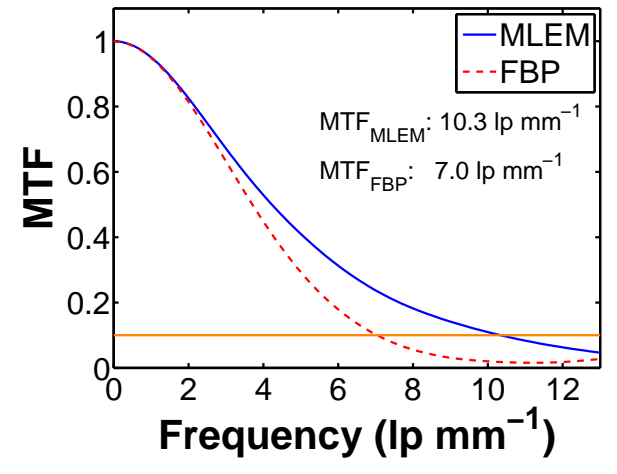

(a) $30 \mu \mathrm{m}$.

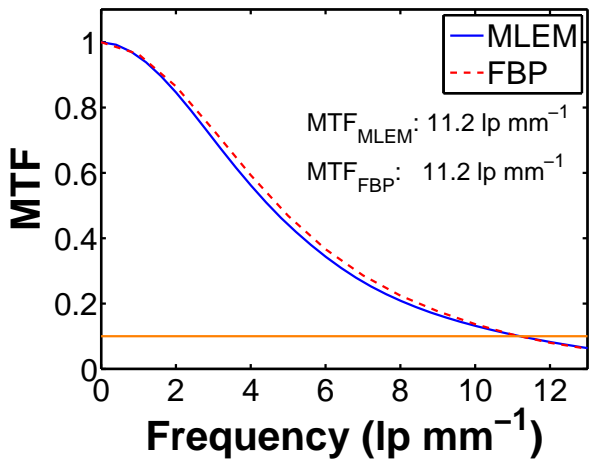

(b) $5 \mu \mathrm{m}$.

Figure 1. Comparison of the performance of spatial resolution for $30 \mu \mathrm{m}$ (a) and $5 \mu \mathrm{m}$ (b) voxel sizes. The frequencies at which the MTF curve is $10 \%$ of its maximum value are reported in each plot.

considered, because the contribution of the scanner's intrinsic spatial resolution $(\sim 90 \mu \mathrm{m})$ is the dominant effect.

Contrast and noise performance achieved with FBP and MLEM were compared with a PMMA phantom $55 \mathrm{~mm}$ in diameter with five holes of $8 \mathrm{~mm}$ axially drilled at $16 \mathrm{~mm}$ off the center. The holes were filled with inserts of synthetic materials modeling fat (polyethylene), soft tissue (PMMA), organs (polyoxymethylene) and soft bone (ploytetrafluoroethylene). The last hole was left empty in order to model air regions inside the body. The average $\left(A_{I}\right)$ and standard deviation $\left(\sigma_{I}\right)$ values in five $4.5 \mathrm{~mm}$ in diameter and $20 \mathrm{~mm}$ height cylindrical volumes of interest (VOI) centered in each insert were considered to calculate the figures of merit described in (3.1) and (3.2). The percent standard deviation (STD) and contrast to noise ratio (CNR) have been considered in the assessment of image noise and the ability in the identification of each material, respectively. The average $\left(A_{B}\right)$ and standard deviation $\left(\sigma_{B}\right)$ in the background in (3.2) correspond to those measured in the soft tissue insert.

$$
\begin{aligned}
\mathrm{STD}_{I} & =\frac{\sigma_{I}}{A_{I}} 100 \\
\mathrm{CNR}_{I} & =\frac{2 \cdot\left|A_{I}-A_{B}\right|}{\sigma_{I}+\sigma_{B}}
\end{aligned}
$$

The noise performance of the MLEM $(0.8 \mathrm{~mm})$, using cubic voxels of $0.8 \mathrm{~mm}$, was compared with the FBP considering $0.8 \mathrm{~mm}$ and $0.4 \mathrm{~mm}$ voxel sizes. The FBP $(0.4 \mathrm{~mm})$ configuration was chosen because yields to images with spatial resolution comparable to MLEM (0.8 mm). Noise (STD) dependence on the number of projections has been evaluated in the fat (figure 2(a)) and organs (figure 2(b)) equivalent tissues. Whatever the considered tissue, similar STD values were obtained with the MLEM and FBP when using $0.8 \mathrm{~mm}$ voxels. The CNR values of air, fat, organs and soft bone respect to soft tissue obtained with the MLEM $(0.8 \mathrm{~mm})$ and FBP $(0.4 \mathrm{~mm})$ are compared in figure 2(c). As we consider the absolute difference in CNR, the values for the air and soft bone respect to soft tissue are similar, this explain the closeness of the curves in figure 2(c). The same occurs with the fat and the organs curves. The smaller STD values achieved with the MLEM algorithm lead to an improvement in CNR values in comparison to those measured with the FBP. The CNR curves of the air and soft bone intersect when the MLEM reconstruction is 


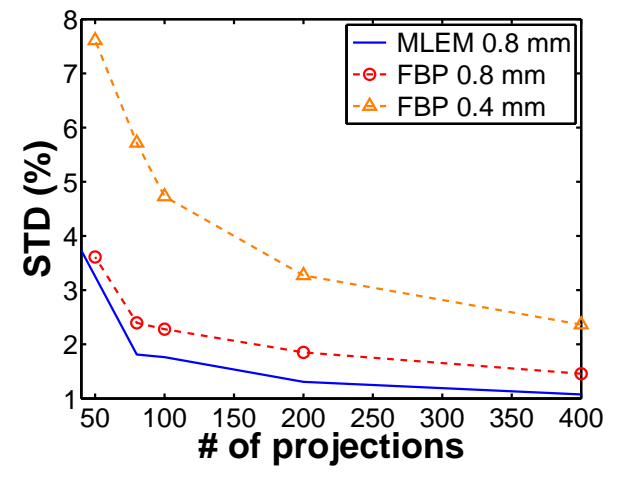

(a) Fat tissue.

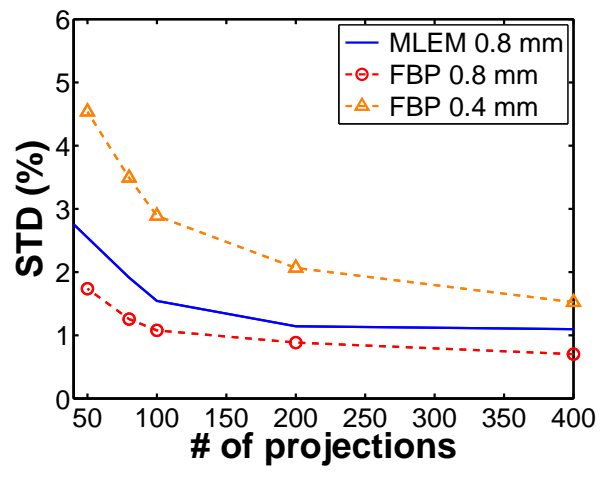

(b) Organs tissue.

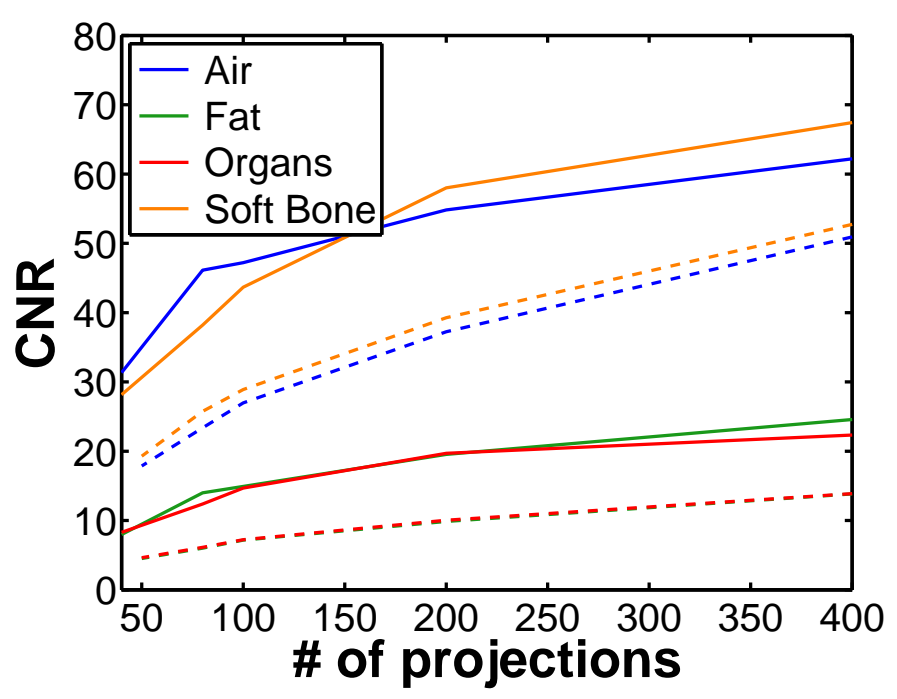

(c) CNR.

Figure 2. Noise (STD) and CNR performance versus the number of projections. STD in the (a) fat and (b) organs tissue equivalent inserts. (c) CNR measured with the MLEM using voxels of $0.8 \mathrm{~mm}$ (solid) and the FBP considering voxels of $0.4 \mathrm{~mm}$ (dashed).

considered, causing that CNR values measured in the air are better than those in soft bone when a small number of projections is employed, while the opposite scenario is observed if the number of projections rises. This exchange in the CNR of air and soft bone is due to the lack of ability of the MLEM to produce sharp transitions between the air insert and its surrounding background, that leads to an overestimation of the STD in the air insert. Therefore, limiting the improvement of CNR values as the number of projections increases.

The performance of the FBP and the MLEM algorithms has also been evaluated in actual acquisitions of a mouse, shown in figure 3. Structures are better defined in the MLEM image (figure 3(b)) than in the FBP one (figure 3(a)) despite the same voxel size has been considered in both cases. The MLEM (figure 3(b)) achieves an image detail comparable to that obtained with the FBP, but using a smaller voxel size (figure 3(c)). Similar behavior is appreciated in the three attenuation profiles shown in figure 4. In all three profiles is observed that the MLEM using a voxel size of $0.8 \mathrm{~mm}$ reproduces the small structures in the air gaps and backbone as accurately as 


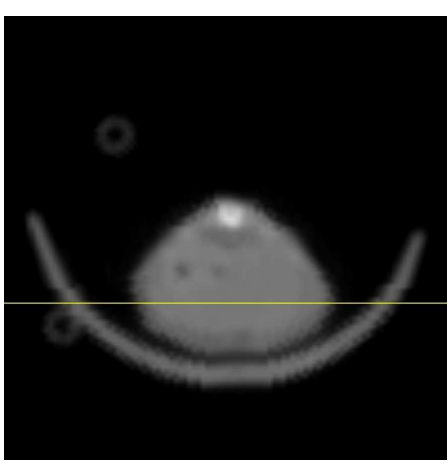

(a) $\operatorname{FBP}(0.8 \mathrm{~mm})$

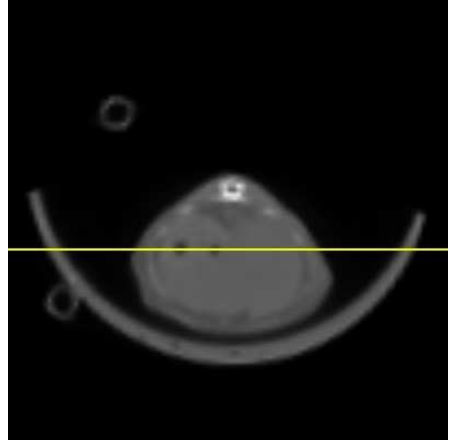

(b) $\operatorname{MLEM}(0.8 \mathrm{~mm})$

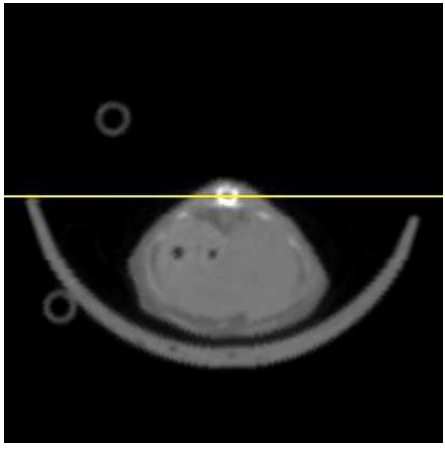

(c) $\operatorname{FBP}(0.4 \mathrm{~mm})$

Figure 3. Transverse view of the abdomen of a mouse reconstructed with (a) the FBP algorithm and a voxel size of $0.8 \mathrm{~mm}$, (b) the MLEM using a $0.8 \mathrm{~mm}$ voxel size and (c) the FBP with a $0.4 \mathrm{~mm}$ voxel size.

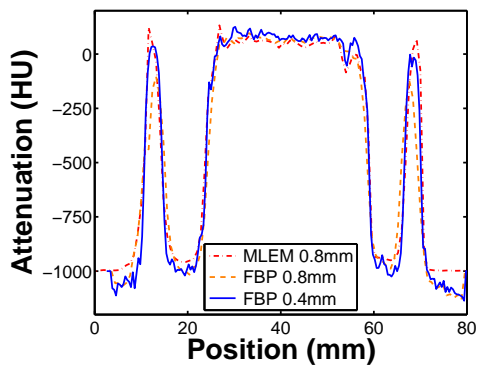

(a)

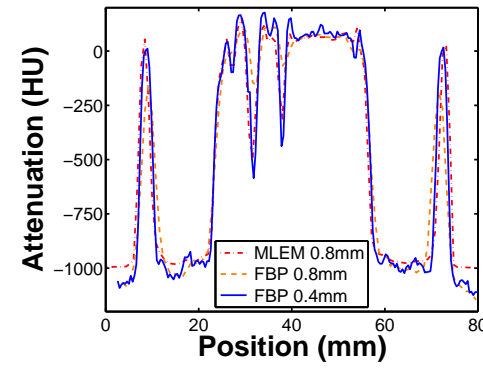

(b)

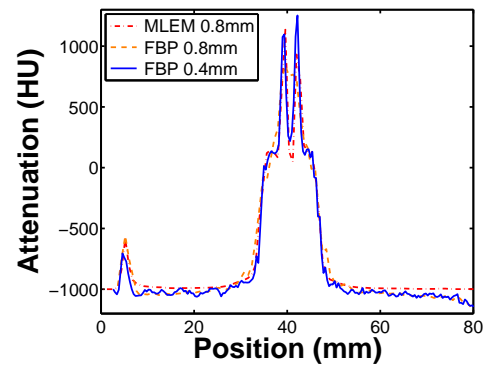

(c)

Figure 4. Comparison of the attenuation profiles obtained with the FBP and the MLEM algorithms along the lines in yellow in figure 3. (a) Attenuation profiles along a uniform region of the abdomen (yellow line in figure 3(a)), (b) profiles along the abdominal region with two air regions (yellow line in figure 3(b)), (c) attenuation profiles crossing the backbone of the mice (yellow line in figure 3(c)).

the FBP using a $0.4 \mathrm{~mm}$ voxel size, while maintaining the less noisy properties of the FBP using a $0.8 \mathrm{~mm}$ in uniform regions like the abdominal tissue.

\section{Conclusion}

Polar symmetry reduces the size of the system matrix in cone beam CT, and overcomes the limitation of a cartesian approach in the iterative reconstruction of clinical images of small animals with voxel sizes smaller than $0.4 \mathrm{~mm}$ using custom computing resources. The MTF curves obtained with the MLEM outperform those measured with the FBP, unless the scanner's intrinsic spatial resolution represents the major contribution. The MLEM $(0.8 \mathrm{~mm})$ yields to images with noise properties similar to the FBP $(0.8 \mathrm{~mm})$. The noisier images in the FBP $(0.4 \mathrm{~mm})$ lead to lower CNR respect to MLEM $(0.8 \mathrm{~mm})$, despite they both produce images with comparable spatial resolution. Thus, MLEM outperforms FBP as long as reconstruction time is not critical. The results obtained with synthetic phantoms have been reproduced with clinical images of a small mouse. Iterative reconstruction improves the image detail without compromising the image noise for a given voxel size. 


\section{Acknowledgments}

This work was supported by the Spanish Plan Nacional de Investigación Científica, Desarrollo e Innovación Tecnológica (I+D+I) under Grant No. FIS2010-21216-CO2-01 and Valencian Local Government under Grants PROMETEO/2008/114, ISIC/2012/013 and APOSTD/2010/012.

\section{References}

[1] L.A. Feldkamp, L.C. Davis and J.W. Kress, Practical cone-beam algorithm, J. Opt. Soc. Am. 1 (1984) 612.

[2] L.A. Sheep and Y. Vardi, Maximum likelihood reconstruction for emission tomography, IEEE Trans. Med. Imaging 1 (1982) 113.

[3] J. Bian et al., Evaluation of sparse-view reconstruction from flat-panel-detector cone-beam CT, Phys. Med. Biol. 55 (2010) 6575.

[4] E.Y. Sidky and X. Pan, Image reconstruction in circular cone-beam computed tomography by constrained, total-variation minimization, Phys. Med. Biol. 53 (2008) 4777.

[5] A.K. Hara et al., Iterative reconstruction technique for reducing body radiation dose at CT: feasibility study, Am. J. Roentgenol. 193 (2009) 764 [Erratum ibid. 193 (2009) 1190].

[6] M.J. Rodríguez-Alvarez, F. Sánchez, A. Soriano, A. Iborra and C. Mora, Exploiting symmetries for weight matrix design in CT imaging, Math. Comput. Model. 54 (2011) 1655.

[7] R.L. Siddon, Fast calculation of exact radiological path for three-dimensional CT array, Med. Phys. 12 (1985) 252.

[8] W. Yao and K. Leszczynski, Analytically derived weighting factors for transmission tomography cone beam projections, Phys. Med. Biol. 54 (2009) 513.

[9] http://www.gem-imaging.com.

[10] A.L.C. Kwan, J.M. Boone, K. Yang and S.-Y. Huang, Evaluation of the spatial resolution characteristics of a cone-beam breast CT scanner, Med. Phys. 34 (2007) 275. 